\title{
Analogue models for FRW cosmologies
}

\author{
Carlos Barceló, , 田 Stefano Liberati, ${ }^{2, \text { 由 }}$ and Matt Visser 3 , 团 \\ ${ }^{1}$ Institute of Cosmology and Gravitation, University of Portsmouth, Portsmouth PO1 2EG, England \\ ${ }^{2}$ Physics Department, University of Maryland, College Park, MD 20742-4111, USA \\ ${ }^{3}$ School of Mathematical and Computing Sciences, Victoria University of Wellington, New Zealand
}

(Dated: 31 March 2003; LATEX-ed August 17, 2018)

\begin{abstract}
It is by now well known that various condensed matter systems may be used to mimic many of the kinematic aspects of general relativity, and in particular of curved-spacetime quantum field theory. In this essay we will take a look at what would be needed to mimic a cosmological spacetime - to be precise a spatially flat FRW cosmology - in one of these analogue models. In order to do this one needs to build and control suitable time dependent systems. We discuss here two quite different ways to achieve this goal. One might rely on an explosion, physically mimicking the big bang by an outflow of whatever medium is being used to carry the excitations of the analogue model, but this idea appears to encounter dynamical problems in practice. More subtly, one can avoid the need for any actual physical motion (and avoid the dynamical problems) by instead adjusting the propagation speed of the excitations of the analogue model. We shall focus on this more promising route and discuss its practicality.
\end{abstract}

PACS numbers: 04.70.Dy, 03.75.Fi, 04.80.-y; gr-qc/0305061

Keywords: Analogue model; cosmology; Bose-Einstein condensate

This essay received an "honorable mention" in the 2003 Essay Competition of the Gravity Research Foundation.

\section{MOTIVATION}

It is by now well known that various condensed matter systems may be used to mimic many of the kinematic aspects of general relativity, and in particular of curved-spacetime quantum field theory [1, 2, 3, 4]. Prior work has focussed largely on black holes and event horizons, but there are also interesting cosmological issues that can be addressed. In this essay we will take a look at what would be needed to mimic a cosmological spacetime - to be precise a spatially flat FRW cosmology - in one of these analogue models. There are two quite different ways of trying to achieve the same goal:

- One might rely on an explosion, physically mimicking the big bang by an outflow of whatever medium is being used to carry the excitations of the analogue model (see e.g. [5]). Unfortunately, this idea appears to encounter dynamical problems in practice, problems not inherent to the type of geometry being reproduced.

- More subtly, one can avoid the need for any actual physical motion (and avoid the dynamical problems) by instead adjusting the propagation speed of the excitations in the analogue model.

The physical metric we will be trying to emulate is that of a spatially flat FRW cosmology

$$
\mathrm{d} s_{\mathrm{FRW}}^{2}=-c^{2} \mathrm{~d} t^{2}+a(t)^{2} \mathrm{~d} \vec{x}^{2},
$$

where $a(t)$ is the scale factor of the universe as a function of time. In contrast the analogue models generically provide effective metrics of the form [1, 2, 3, 4]

$$
\mathrm{d} s_{\text {effective }}^{2}=\frac{\rho(t, \vec{x})}{c_{s}(t, \vec{x})}\left\{-\left[c_{s}^{2}(t, \vec{x})-v^{2}(t, \vec{x})\right] \mathrm{d} t^{2}-2 \vec{v}(t, \vec{x}) \cdot d t \mathrm{~d} \vec{x}+\mathrm{d} \vec{x}^{2}\right\} .
$$

Here $\vec{v}(t, \vec{x})$ is the physical velocity of the medium, $c_{s}(t, \vec{x})$ is the propagation speed of whatever excitations we are interested in studying, and the conformal prefactor $\rho(t, \vec{x}) / c_{s}(t, \vec{x})$ depends both on the dimensionality of spacetime

\footnotetext{
*carlos.barcelo@port.ac.uk http://www.tech.port.ac.uk/staffweb/barceloc/

†liberati@physics.umd.edu http://www2.physics.umd.edu/ liberati

'Matt.Visser@mcs.vuw.ac.nz http://www.mcs.vuw.ac.nz/ visser
} 
and [to some extent] on the specific choice of the analogue model. In the form presented above the conformal factor is appropriate to either ordinary sound in a classical fluid [1, 2, 3, 4], or to phonons in a BEC [6], both in $(3+1)$ dimensions.

We now want to consider the fundamental question - given the availability of "effective metrics" of type (2), how close can one get to reproducing a FRW metric of type (1). That this question is non-vacuous can be deduced from the observation that in the black hole context "effective metrics" of type (2) never exactly reproduce the Schwarzschild geometry [3], they can do so only up to an overall conformal factor. We wish to check, in particular, if the same phenomenon shows up in a cosmological context.

\section{EXPLOSIONS}

As mentioned in the introduction, one plausible approach is to rely on an actual physical explosion in the medium to mimic the big bang [5]. Start with the FRW metric (1) and substitute

$$
\vec{z}=b(t) \vec{x} ; \quad \mathrm{d} \vec{z}=b(t) \mathrm{d} \vec{x}+\dot{b}(t) \vec{x} \mathrm{~d} t ; \quad b(t) \mathrm{d} \vec{x}=\mathrm{d} \vec{z}-\frac{\dot{b}(t)}{b(t)} \vec{z} \mathrm{~d} t
$$

Then, introducing a Hubble-like parameter,

$$
H_{b}(t)=\frac{\dot{b}(t)}{b(t)}
$$

the FRW metric (11) is transformed to

$$
\mathrm{d} s_{\mathrm{FRW}}^{2}=-\left[c^{2}-\frac{a^{2}}{b^{2}} H_{b}(t)^{2} \vec{z}^{2}\right] \mathrm{d} t^{2}-2 \frac{a^{2}}{b^{2}} H_{b}(t) \vec{z} \cdot \mathrm{d} \vec{z} \mathrm{~d} t+\frac{a^{2}}{b^{2}} \mathrm{~d} \vec{z}^{2} .
$$

This metric is equivalent to the required "effective metric" form [equation (2)], provided we take

$$
\vec{v}(t, \vec{z}) \leadsto H_{b}(t) \vec{z} ; \quad c_{s} \leadsto \frac{b}{a} c ; \quad \frac{\rho}{c_{s}} \leadsto \frac{a^{2}}{b^{2}} .
$$

The continuity equation for the medium,

$$
\dot{\rho}+\vec{\nabla} \cdot(\rho \vec{v})=0
$$

particularized to the flow field $\vec{v}(t, \vec{z}) \rightsquigarrow H_{b}(t) \vec{z}$ implies

$$
\dot{\rho}+3 \rho H_{b}(t)=0 ; \quad \Rightarrow \quad \rho(t) \propto \frac{1}{b^{3}(t)} .
$$

Using the last equivalence in Eq. (6) we can now fix the behaviour of $c_{s}(t)$ as a function of $a(t)$ and $b(t)$

$$
c_{s}(t) \propto \frac{1}{a(t)^{2} b(t)}
$$

Equations (8) and (9), together with the first equivalence in Eq. (6), completely fix the relation between the hydrodynamical quantities $\left(\rho(t), \vec{v}(t, \vec{z}), c_{s}(t)\right)$ and the cosmological solution parameters $(a(t), b(t), c)$.

Although the "explosion route" just discussed seems promising, one should note that because of the linearly rising velocity field $\vec{v}=H_{b} \vec{z}$, this particular realization of a FRW effective geometry is guaranteed to possess an apparent horizon, a spherical surface in which the speed of the fluid surpasses the speed of sound. From a dynamical point of view, this might introduce many practical problems not intrinsically inherent to the type of geometries we are trying to reproduce. In this sense, we view the use of an exploding medium as not being a particularly useful analogue for an expanding FRW universe. 


\section{VARYING PROPAGATION SPEED}

A much better analogue of an expanding FRW universe can be obtained by keeping the medium at rest and instead varying the propagation speed of the excitations. In this case the continuity equation implies that $\rho(t, \vec{x})$ is a constant, so that we can rescale the metric by a constant factor $c_{0} / \rho$ (where $c_{0}$ is any convenient reference speed) obtaining

$$
\mathrm{d} s_{\text {effective }}^{2}=\frac{c_{0}}{c_{s}(t)}\left\{-c_{s}^{2}(t) \mathrm{d} t^{2}+\mathrm{d} \vec{x}^{2}\right\}=-c_{0} c_{s}(t) \mathrm{d} t^{2}+\frac{c_{0}}{c_{s}(t)} \mathrm{d} \vec{x}^{2}
$$

Now introduce a pseudo-time $\tau$, related to laboratory time via $\mathrm{d} \tau=\mathrm{d} t \sqrt{c_{s}(t) / c_{0}}$. Then

$$
\mathrm{d} s_{\text {effective }}^{2}=-c_{0}^{2} \mathrm{~d} \tau^{2}+\frac{c_{0}}{c_{s}(t)} \mathrm{d} \vec{x}^{2} .
$$

Consequently we completely reproduce a FRW cosmology provided we identify

$$
c_{0} \mathrm{~d} \tau \leadsto c \mathrm{~d} t_{\mathrm{FRW}} ; \quad \frac{c_{0}}{c_{s}(t)} \leadsto a(\tau)^{2} .
$$

That is, an expanding universe corresponds in the effective geometry to a decreasing propagation speed. A tricky point is that $c_{s}$ is still presented in terms of laboratory time $t$ as the speed $\|\mathrm{d} \vec{x} / \mathrm{d} t\|$. However in terms of the pseudo-time $\tau$ the excitations propagate at speed

$$
\bar{c}_{s}(\tau)=\left\|\frac{\mathrm{d} \vec{x}}{\mathrm{~d} \tau}\right\|=\frac{\mathrm{d} t}{\mathrm{~d} \tau}\left\|\frac{\mathrm{d} \vec{x}}{\mathrm{~d} t}\right\|=\sqrt{\frac{c_{0}}{c_{s}(t)}} c_{s}(t)=\sqrt{c_{0} c_{s}(t)} .
$$

Having obtained the general analogue of the flat FRW metric it is interesting to investigate the analogue equivalent of the inflationary solution. We can start by re-writing the analogue Hubble factor as

$$
H=\frac{a^{\prime}(\tau)}{a(\tau)}=\frac{1}{2} \sqrt{\frac{c_{s}}{c_{0}}} \dot{a} \frac{\mathrm{d} t}{\mathrm{~d} \tau}=-\frac{1}{2} \sqrt{\frac{c_{0}}{c_{s}}} \frac{\dot{c_{s}}}{c_{s}} .
$$

where the prime represents derivative with respect to the pseudo-time and the dot with respect to the laboratory time. The inflationary solution is then easily obtained by using a exponential law in pseudo-time $\tau$

$$
a(\tau)=e^{H \tau} ; \quad a(t)=H t ;
$$

corresponding to a power law in physical time for the speed of sound (measured in physical time)

$$
c_{s}(t)=\frac{c_{0}}{a^{2}(\tau)}=\frac{c_{0}}{H^{2} t^{2}}
$$

In summary the nice feature of the variable propagation speed route to a FRW analogue is that it is relatively clean; there is no moving medium which might impact the physical boundaries of any experimental apparatus and one can instead focus on what we feel is the central issue - how to physically manipulate the propagation speed $c_{s}$ (or its pseudo-time equivalent $\bar{c}_{s}$ ).

\section{SUITABLE PHYSICAL MECHANISM: FESHBACH RESONANCE IN A BEC}

From the preceding discussion it is clear that one attractive route to simulating a FRW cosmology in an effective geometry is by rapid manipulation of the propagation speed of whatever excitations we might wish to focus attention on. But how is that to be accomplished? There is one particular medium, currently the center of much experimental interest, for which an appropriate mechanism has been demonstrated to exist. Here we are referring tho the use of Feshbach resonances [7] in Bose-Einstein condensates [BECs].

BECs are promising analogue systems of gravity. They have been extensively studied [4, 6, 8, 9] in relation to the possibility of simulating black hole geometries and Hawking radiation. The basic equation used in describing the condensate is the Gross-Pitaeveskii [GP] equation

$$
-\mathrm{i} \hbar \frac{\partial}{\partial t} \psi(t, \mathbf{x})=\left(-\frac{\hbar^{2}}{2 m} \nabla^{2}+V_{\mathrm{ext}}(\mathbf{x})+\kappa|\psi(t, \mathbf{x})|^{2}\right) \psi(t, \mathbf{x}) ; \quad \kappa=\frac{4 \pi \sigma \hbar^{2}}{m}
$$


Here $\psi(x, t)$ is the (classical) wave function of the condensate, $\left(|\psi(x, t)|^{2}=n=\right.$ the particle density of the condensate), $V_{\text {ext }}$ is the trapping potential and $\sigma$ is the $s$-wave scattering length for the atoms (which have mass $m$ ). [Actually it is conventional to use the symbol $a$ for the scattering length of a BEC condensate. We adopt this unusual notation to avoid any confusion with the scale factor of the FRW metric.] The generic analogue model can be easily be obtained by considering the propagation of excitations in the condensate. These are described by a wave function which closely resembles that of a scalar field in a curved spacetime described by the metric (2) with $\rho$ replaced by $n m$ and where $c_{s}^{2}=\kappa n / m$ (see [6] for a detailed derivation). The key point is that the propagation speed is proportional to the scattering length

$$
c_{s}^{2} \propto \sigma
$$

Let us now qualitatively explain we can simulate cosmological expansion within this model. We start with a condensate in a stationary state described by a constant background density in a sufficiently large volume. This is a solution of the Gross-Pitaevskii equation. For this, one needs to have a potential that reproduces a sufficiently large hard-walled box. Using a Feshbach resonance it is now possible to change at will the scattering length $\sigma$ in the condensate. If one now decreases the value of the scattering length in a sufficiently slow manner (the timescale will be discussed below), then at the same time the value of the speed of sound will decrease. The analysis of the previous Section then shows that this leads to an effective FRW geometry. Fluctuations of the condensate will propagate in an effective metric which is an analogue to a spatially flat FRW geometry.

A subtle point is that when the time dependence is introduced in the system by a time-varying scattering length one must be sure to work in a regime where the background configuration is "instantaneously" reacting to theses changes. Thus we must assure that the GP equation (from which the analogue gravity framework is derived) holds at each instant of time. The validity of the GP in describing the Bose-Einstein condensate is related to the validly of several crucial assumptions, generally stated to be the "mean-field" approximation and the dilute gas approximation. It is nevertheless important to note that in a dynamical situation a third approximation, which we can call "Markovian" approximation, is implicitly assumed.

The Markovian approximation is related to the fact that in dynamical situations the two-body time-dependent scattering matrix can have a complicated form due to the "memory" of the system. In these situations the system is described by a GP-like equation where the interaction term includes a "delay term" described by an integration over time. The necessary assumption in order to have a Markovian description of the dynamics (which together with the two previous approximations leads to the GP equation) is then that the timescales on which external parameters are changing are longer that the two-body collisional duration. That is, longer than the timescale over which a single interaction happens. (Reduced to the bare bones, we are asking that the scattering length does not change significantly during the period when a pair of atoms are interacting.)

We can estimate the two-body collision time by a simple calculation. All we need is the typical size of the region of strong interaction of two atoms in the condensate, and the typical speed with which they move. The first quantity can be assumed to be of the order of the Van der Waals length. This length is basically the size of the region of strong interaction: For $r \ll \lambda_{v d W}$ the scattering wave function oscillates rapidly due to the strong interaction potential. In alkali ground state interactions, $\lambda_{v d W}$ is typically of order of few nanometers, $\lambda_{v d W} \approx 1 \mathrm{~nm}$.

Regarding the typical speed of the atoms, this is set by the de Broglie momentum generated by the trap confinement: $p=h / R$ and $\bar{v}=p / m$. We shall assume a trap of typical size of ten microns. For a typical Bose-Einstein condensate one then gets an interaction timescale

$$
t_{i}=\frac{\lambda_{v d W}}{\bar{v}}=\frac{\lambda_{v d W} m R}{h} \approx 10^{-6} \mathrm{~s},
$$

so it would appear that a microsecond is the shortest timescale allowed for the change in $\sigma$.

\section{ANALOGUE COSMOLOGICAL PARTICLE CREATION}

Now that the above analysis has shown that there is a regime for which a varying scattering length can be used to simulate a FRW-like effective metric one may wonder about the behaviour of the fluctuations on such a time-dependent background.

The equation satisfied by the quantum fluctuations is, in the acoustic approximation (that is for long wavelengths), that of a massless scalar field over an expanding background and therefore, it will lead to cosmological pair production of particles. Interestingly it was recently demonstrated [10] that not all analogue models of gravity are suitable for simulating particle creation from the quantum vacuum. Indeed, it may happen that even if the classical equation for the perturbations on the background resembles that for a field in curved spacetime, nonetheless one might fail to 
mimic quantum particle creation because the commutation relations of the analogue field are not the correct one for the physical quantum field. In this regard it is useful to note that it can be explicitly shown [10] that in the case of BECs the structure of the commutator is the correct one.

We then expect to be able to produce quantum excitations with several frequencies, typically determined by the rapidity of the change in the scattering length. In particular if $t_{\min }$ is the shortest timescale over which we can physically drive the system then $\nu_{\text {peak }} \approx 1 / t_{\min }$ is the peak frequency of the created quasi-particles spectrum. It is also important to check that this frequency corresponds to a wavelength shorter than the physical size of the condensate $R$. An easy way to do this is to confront the interaction time $t_{i}$ given in Eq. (19) with the crossing time of the condensate, $t_{\mathrm{size}}=R / c$.

$$
\frac{t_{i}}{t_{s i z e}}=\frac{\lambda_{v d W} m c}{h}=\frac{2 \pi \lambda_{v d W}}{\xi}
$$

where we have introduced the healing length of the condensate, defined to be $\xi=\hbar /\left(m c_{s}\right)$. For typical BEC system $\xi \approx 0.1-10 \mu \mathrm{m}$ (assuming an average value of the scattering length of a few nanometers) so

$$
\frac{t_{i}}{t_{\text {size }}} \approx 10^{-2}-10^{-4}
$$

Thus there is a viable window of timescales (for the time dependence of the scattering length) for which both the GP equation holds, and the quasi-particles produced have wavelengths shorter than the physical size of the condensate. The possibility of simulating cosmological particle creation is a significant advance that deserves further investigation, and might be remarkably important in the future. The simulation of inflationary scenarios, and the relevant particle creation, could lead to a better understanding of the generation of primordial inflationary perturbations and their role in the generation of large scale structure. Moreover it should be noted that the quasi-particles generated in this way will be characterized by a phononic dispersion relation $\omega^{2}=c_{s}^{2} k^{2}$ only at long wavelengths with respect to the healing length of the condensate. In general for wavelengths comparable with the healing length of the condensate the Bogoliubov dispersion relation

$$
\omega=\sqrt{c_{s}^{2} k^{2}+\left(\frac{\hbar}{2 m} k^{2}\right)^{2}},
$$

will hold [1]. This is particularly interesting due to the recent intense debate about the detectability of inflationary spectrum due to these kind of dispersion relations [12, 13].

\section{CONCLUSIONS AND PROSPECTS}

Our major conclusions are four-fold:

- At a theoretical level, it is clear that any mechanism for changing the propagation speed in a stationary medium is from a mathematical perspective equivalent to working in an expanding (or collapsing) spatially flat FRW universe.

- At an experimental level, the use of a Feshbach resonance in a Bose-Einstein condensate yields a way of influencing the scattering length, and hence the propagation speed, without changes in condensate density - and more importantly without violating the approximations made in deriving the Gross-Pitaevskii equation on which the entire "effective metric" approach to phonon propagation in BECs is based.

- At a very practical engineering level, the relevant parameters seem to be well within our technological horizon.

- Building an analogue FRW cosmology (suitable for testing semiclassical quantum effects) seems considerably less problematic than building an analogue black hole [4, [8, 9].

In summary: The prospects for direct laboratory simulation of an expanding universe, and consequent cosmological particle production are very good. Given the relatively small number of experimental tests of curved space quantum field theory, any progress along these lines is important. Our long range goal is to turn at least some aspects of cosmology into a laboratory science, not just an observational science.

[1] W. G. Unruh, "Experimental Black Hole Evaporation," Phys. Rev. Lett. 46 (1981) 1351. 
[2] M. Visser, "Acoustic Propagation In Fluids: An Unexpected Example Of Lorentzian Geometry," arXiv:gr-qc/9311028

[3] M. Visser, "Acoustic black holes: Horizons, ergospheres, and Hawking radiation," Class. Quant. Grav. 15 (1998) 1767 arXiv:gr-qc/9712010.

[4] M. Novello, M. Visser and G. Volovik, "Artificial Black Holes," (World Scientific, 2002).

[5] P. O. Fedichev and U. R. Fischer, "'Cosmological' particle production in oscillating ultracold Bose gases: The role of dimensionality", arXiv:cond-mat/0303063

Ibidem, "Hawking radiation from sonic de Sitter horizons in expanding Bose-Einstein-condensed gases", arXiv:cond-mat/0304342

[6] C. Barceló, S. Liberati and M. Visser, "Analog gravity from Bose-Einstein condensates," Class. Quant. Grav. 18 (2001) 1137 arXiv:gr-qc/0011026.

[7] S. L. Cornish, N. R. Claussen, J. L. Roberts, E. A. Cornell, C. E. Wieman, Stable 85Rb Bose-Einstein Condensates with Widely Tunable Interactions Phys. Rev. Lett 85, 1795 (2000). cond-mat/0004290

J. L. Roberts, N. R. Claussen, S. L. Cornish, E. A. Donley, E. A. Cornell, C. E. Wieman Controlled Collapse of a Bose-Einstein Condensate cond-mat/0102116

Elizabeth A. Donley, Neil R. Claussen, Simon L. Cornish, Jacob L. Roberts, Eric A. Cornell, Carl E. Wieman Dynamics of collapsing and exploding Bose-Einstein condensates cond-mat/0105019

[8] L. J. Garay, J. R. Anglin, J. I. Cirac and P. Zoller, "Black holes in Bose-Einstein condensates," Phys. Rev. Lett. 85 (2000) 4643 arXiv:gr-qc/0002015.

L. J. Garay, J. R. Anglin, J. I. Cirac and P. Zoller, "Sonic black holes in dilute Bose-Einstein condensates", Phys. Rev. A 63 (2001) 023611 arXiv:gr-qc/0005131.

[9] C. Barceló, S. Liberati and M. Visser, "Towards the observation of Hawking radiation in Bose-Einstein condensates," arXiv:gr-qc/0110036

[10] W. G. Unruh and R. Schutzhold, "On slow light as a black hole analogue," arXiv:gr-qc/0303028

[11] M. Visser, C. Barceló and S. Liberati, "Acoustics in Bose-Einstein condensates as an example of broken Lorentz symmetry," arXiv:hep-th/0109033

[12] R. H. Brandenberger, "Trans-Planckian physics and inflationary cosmology," arXiv:hep-th/0210186

J. Martin and R. H. Brandenberger, "The Corley-Jacobson dispersion relation and trans-Planckian inflation," Phys. Rev. D 65 (2002) 103514 arXiv:hep-th/0201189.

[13] J. C. Niemeyer, R. Parentani and D. Campo, "Minimal modifications of the primordial power spectrum from an adiabatic short distance cutoff," Phys. Rev. D 66 (2002) 083510 arXiv:hep-th/0206149. 